\title{
Discrete Particle Swarm Optimization Algorithms for the Prize-Collecting Call Control Problem on Lines
}

\author{
Qian-Na Cui \\ School of Mathematics and Statistics, Yunnan University \\ Kunming, 650500, P.R.China \\ cuiqianna@126.com
}

Xing Wu

School of Mathematics and Statistics, Yunnan University Kunming, 650500, P.R.China

wuxing123@163.com

\author{
Qiao-Yan Zhu \\ School of Mathematics and Statistics, Yunnan University \\ Kunming, 650500, P.R.China \\ zqy12356@qq.com
}

\begin{abstract}
In this paper,we propose two discrete particle swarm optimization algorithms to solve a prize-collecting call control problem on lines based on the classical particle swarm optimization algorithm. The two algorithms combine simulated annealing and genetical methods with particle swarm optimization respectively. Then, we do lots of experiments to compare the two discrete particle swarm optimization algorithms we addressed, in which we compare the results and iteration time and the convergence of two algorithms under some given variables. Finally, computational results show that the proposed discrete particle swarm optimization with genetic algorithm is very efficient and can be quickly obtained good results.
\end{abstract}

Keywords-DPSO algorithm; prize-collecting; call control; lines

\section{INTRODUCTION}

In recent years, much attention has been paid to the unsplittable flow problem (UFP). Simply summarized the definition of this problem: given an undirected graph where each edge has its capacity and a set of vertex pairs with a positive demand and a positive profit. The goal is to find a subset with the maximum profit form the given set so that each chosen pair from the subset can satisfy its full demand without exceeding its capacity.

Bansal et al. [1] considered the UFP on a line, they simply introduced that the UFP is NP-hard. To solve this problem, they added dynamic programming into linear programming relaxation to avoid a $\Omega(n)$ integrality gap generated by linear programming. They presented the first polynomial time $O(\log n)$ approximation algorithm for the problem without any assumptions, which broke the previous conclusion that is no polynomial time $O(n)$ approximation algorithm was known in the past.
Bonsma et al. [2] studied the UFP on a path, they presented a constant-factor $(7+\varepsilon)$ approximation algorithm for this problem, which improved on the previous ratio of $O(\log n)$. They also showed a $2+\varepsilon$ -approximation algorithm with slightly violated the capacities, then they proved that this problem is strongly NP-hard. Based on their results, another group gave a new improvement. Anagnostopoulos et al. [3] implied a $2+\varepsilon$ approximation for the same problem without violating any capacities, which was better than the $7+\varepsilon$ ratio for any constant $\varepsilon>0$. Batra et al. [4] studied the same problem, they obtained a PTAS when the the ratio of a task's profit and demand lie in a constant range. They also obtained a PTAS, in which they were allowed to shorten the paths of the tasks.

UFP was widely used in various fields, for which studies did a lot of researches about its actual problems, such as the problem of resource allocation. Bar-Noy et al.[5] presented approximation algorithms for solving resource allocation and scheduling problems, which factors applied to many problems such as dynamic storage allocation and so on. Li.et al. [7] considered another problem about UFP, which is the ring loading problem with penalty cost. In that paper, they showed a 1.58-approximation algorithm for the demand unsplittable case. Later, motivated by the research of prize-collecting Steiner tree problem [8], Li. et al. [6] introduced the prize-collecting call control (PCCC) problem on lines, for which they designed a 1.58-approximation algorithm using a randomized rounding technique.

In our paper, we do a research about (PCCC) problem on lines proposed by Li. et al. [6], which is prize-collecting call control problem on lines, the description of this problem is as follows. Given an line $G=(V, E)$ and a set of $K$ pairs of vertexes $\mathscr{P}=\left\{P_{k} \mid k=1,2, \ldots, K\right\}$, in which each pair of vertexes is representative as a request path. Each request path $P_{k}$ has a positive demand $d_{k}$ 
and a positive penalty cost $p_{k}$. The objective of PCCC problem is to find a subset $\mathscr{P}^{\prime}$ from $\mathscr{P}$ so that the sum of the total demand $\sum_{k: P_{k} \in \mathscr{P}^{\prime} d_{k}}$ and the total penalty cost

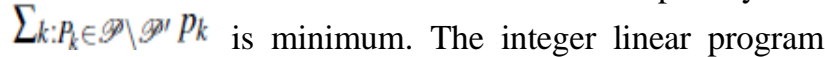
(ILP) of this problem is as follows:

$$
\left\{\begin{array}{l}
\min f=L+\sum_{k=1}^{K} p_{k}\left(1-x_{k}\right) \\
\sum_{\substack{k: P_{k} \in \mathscr{P} \\
x_{k} \in\{0,1\}}} d_{k} x_{k} \leq L
\end{array}\right.
$$

in which for each $P_{k} \in \mathscr{P}, P_{k}$ is either accepted if $x_{k} \equiv 1$ or rejected once $x_{k} \equiv 0$ and $L$ is the maximum load of accepted paths, $f$ is simply represent as the objective function. Li. et al. [6] proved that the PCCC problem on lines is NP-hard.

To solve above problem, we propose two algorithms based on classical particle swarm algorithm. Kennedy and Eberhart [9] proposed particle swarm optimization algorithm in 1995, which is intended to gain a global optimal value. The principles of PSO algorithm can be described as follows [9]. It is assumed that the search space is $n$-dimension and population size is $M$, in which the position and velocity of particle $i$ in the $t$ th iteration is defined by $X_{i}^{t}=\left(x_{i 1}^{t}, x_{i 2}^{t}, \mathrm{~L}, x_{i n}^{t}\right) \quad$ and $V_{i}^{t}=\left(v_{i 1}^{t}, v_{i 2}^{t}, \mathrm{~L}, v_{i n}^{t}\right)$ where $i=1,2, \mathrm{~L}, M$. The flying speed and position of particle $i$ in the $j$ th $(j=1,2, \mathrm{~L}, n)$ subspace of the $t$ th iteration are calculated according to:

$$
\begin{gathered}
v_{i j}^{t+1}=w v_{i j}^{t}+c_{1} r_{1}\left(p_{i j}^{t}-x_{i j}^{t}\right)+c_{2} r_{2}\left(g_{j}^{t}-x_{i j}^{t}\right) \\
x_{i j}^{t+1}=x_{i j}^{t}+v_{i j}^{t+1}
\end{gathered}
$$

where $w$, called weight, is a constant to control the impact of the previous velocities on the current velocity. $c_{1}$ and $c_{2}$ are acceleration factors, which can impact the moving trail of particles, $r_{1}$ and $r_{2}$ are uniformly distributed random variables in $[0,1], g_{j}$ can be representative as the best history position in the whole population, $p_{i j}$ is the best history position of the current particle.

\section{THE ALGORITHM OF DPSO}

Due to the continuous character of PSO, Kashan et al. [10] proposed a discrete particle swarm optimization algorithm for scheduling parallel machines. We use this algorithm methods efficiently to propose two DPSO algorithms and then solve the PCCC problem with them.

\section{A. The Proposed DPSO-GA Algorithm}

We set $k$ numbers of dimensions are presented each for one of $k$ request calls, hence, a solution for the problem is represented by an array whose length is equal to the number of non-zero elements of $k$-dimention array. The location of a particle in the $i$ th dimension represents the $i$ th path to be accepted or rejected, in which the $i$ th path is accepted if the $i$ th value is 1 , or rejected if the if the $i$ th value is 0 . We draw lessons from Kashan et al. [10] and propose a DPSO algorithm with the basis on GA method to solve the PCCC problem on lines, which is called DPSO-GA.

The process of generating a new position for selecting an optimal solution in the given particle swarm is depicted as follows [10]:

$$
\begin{gathered}
V_{i}^{t+1}=V_{i}^{t} \oplus\left(R_{1} \otimes\left(P_{i}^{t} \ominus X_{i}^{t}\right)\right) \oplus\left(R_{2} \otimes\left(P_{g}^{t} \ominus X_{i}^{t}\right)\right) \\
X_{i}^{t+1}=X_{i}^{t} \oplus V_{i}^{t+1}
\end{gathered}
$$

where $R_{1}$ and $R_{2}$ are 1-by- $k$ arrays compromising 0 or 1 elements. These random arrays are generated from a Bernoulli distribution in which the probability of getting 1 is equal to 0.5 . $V_{i}^{t}$ and $X_{i}^{t}$ are the $i$ th particle current velocity and position arrays, respectively. $P_{i}^{t}$ and $P_{g}^{t}$ are the $i$ th particle best position and the global best position in history. The subtraction operator $(\ominus)$ and the multiply operator $(\otimes)$ used by Kashan et al. [10] in equation (3) and (4) are also our operators. The add operator $(\oplus)$ is a crossover operator that method is typically used in genetic algorithms, which method is the only different point with Kashan et al. [10]. First, it randomly generates two cross points from particle chain A. Next, it exchanges the sub-chain between the two points and two new chains obtained. Then, we select one of the new chains randomly to be the result of the add operator.

\section{B. The Proposed DPSO-SA Algorithm}

Simulated Annealing (SA) is a random-search technique proposed by Metropolis [11], whose major advantage is an ability of suddenly jumping with probability to avoid becoming trapped in local minima. So, we can add the SA into DPSO algorithm to solve the PCCC problem on lines efficiently, called DPSO-SA algorithm, it continues the updating equation (3) and (4). Before the updating of the position and the velocity vector, given an initial temperature $T_{0}$, we compute the temperature $T_{i}$ by $e^{-f\left(p_{i}^{t}\right)-f\left(p_{g}^{t}\right) / T(0)}$ [11], the algorithm 
repeats the updating operator until reaching the temperature balance $T_{i}$ [11]. More important, the simulated annealing operator is $T_{i+1}=C T_{i}$ where $C \in(0,1)$ until satisfying the convergence conditions.

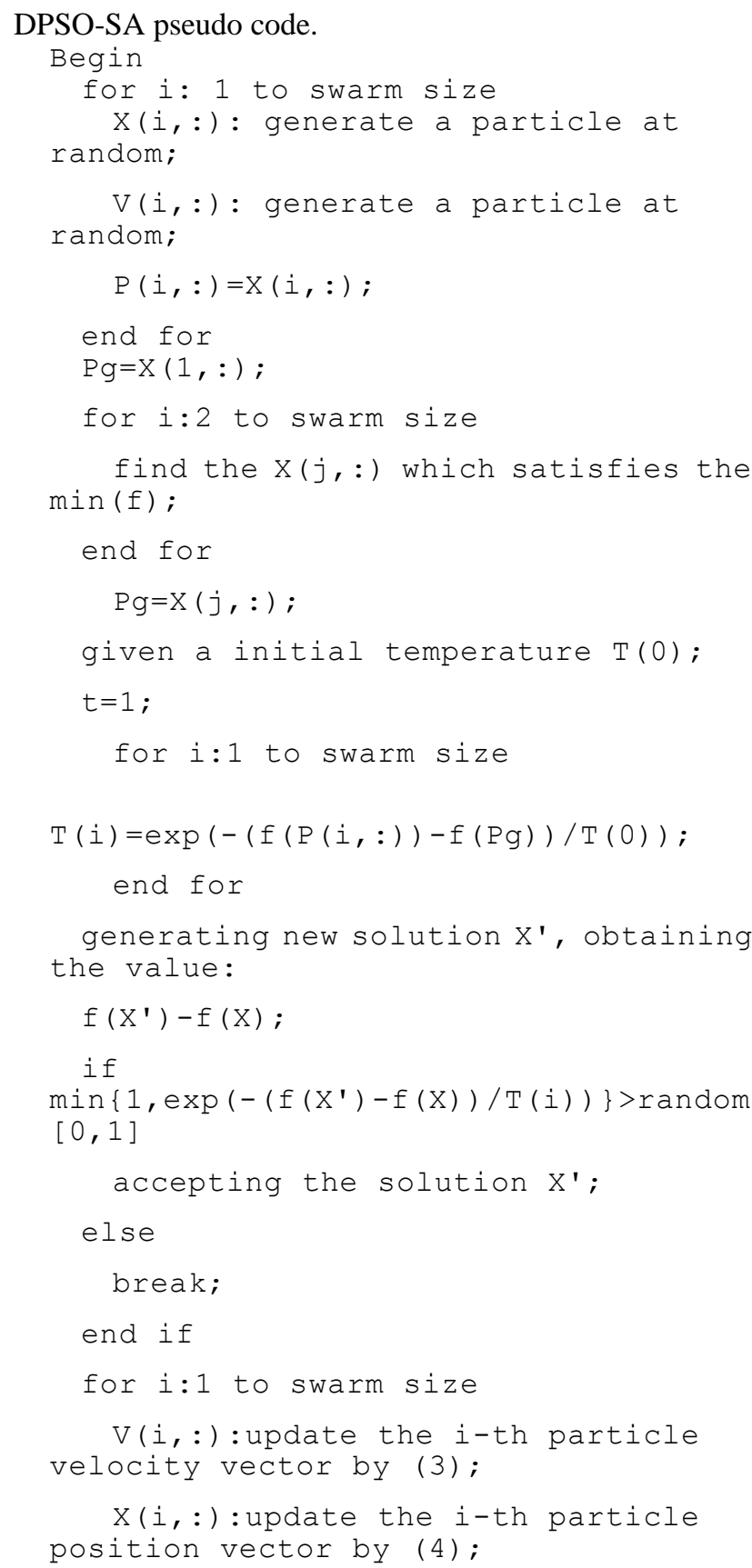

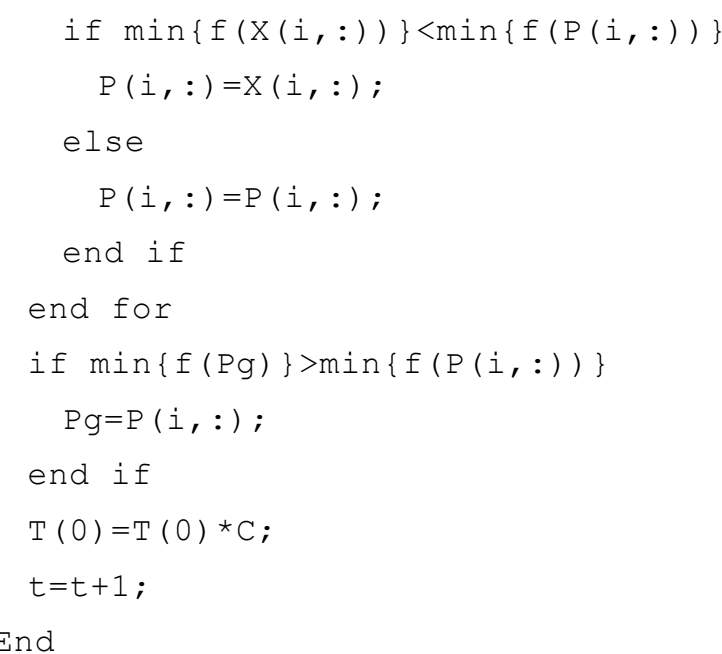

\section{COMPUTATIONAL EXPERIMENTS}

The two DPSO algorithms we proposed are better or not to solve effectively the PCCC problem on lines, we do lots of experiments to prove it. We will give 100 particles to each algorithm and let the two programs run 100 times for each experiment in MATLAB to reach results, and use the finial result and the total running time to compare what we want to. In all following tables, we let "result" and "time" be representatives.

\section{A. Experiments of DPSO-SA}

Due to the optimal value obtained by SA is different when its parameters changed a little, we do some experiments to obtain the best parameters. An experimental frame-works, namely $\mathrm{E} 1$, is considered each of them having two influence factors: the initial temperature $T_{0}$ and the annealing function $T_{i+1}=C T_{i}$. Theoretically, the annealing velocity is no more quicker than $T_{i}=T_{0} /(1+\ln (i))$, in other words, $T_{i}$ is diminishing once $i>2$, so we design $C$ is an alterable constant in $(0,1)$. In order to obtain the best result, insuring the initial temperature big enough is of great importance, then we decide that the initial temperature is similar to the function value of initial global best position $f\left(p_{g}\right)$, that is to say let $T_{0}$ be the relative to the $f\left(p_{g}\right)$, for which we definite $T_{0}=r f\left(p_{g}\right)$ where $r$ is a changeable constant.

TABLE 1. Framework E1

\begin{tabular}{cccccc}
\hline & $K$ & $d_{k}$ & $p_{k}$ & $r$ & $C$ \\
\hline E1 & 100 & $U[1,3]$ & $U[1,5]$ & $0.5,0.7,1.0,1.2,1.5$ & $0.2,0.4,0.5,0.7,0.9$ \\
\hline
\end{tabular}


We can see the experimental results about DPSO-SA with $K=100$ in Table2. Clearly, when the value of $r$ is $0.5,0.7,1.0,1.2,1.5$, the two values change separately. The fluctuating values are $18,8,20,33,16$ and 0.4990 , $0.0760,0.9050,0.8890,0.7950$, so the two vales fluctuate least when $r=0.7$. Similarly, the changeable range is least when $C=0.7$, the two values only change 2 and 0.1410 . In general, the two parameters result into slightly alteration, so we will choose the two best parameters to do the following experiments, that is to say $r=0.7$ and $C=0.7$.

\section{B. Contrastive Experiments between DPSO-GA and DPSO-SA}

Analysis of results and time. In this part, we study the comparison on the effectiveness of the DPSO-GA algorithm and the DPSO-SA algorithm by do a mount of experiments. Three experimental frameworks, namely E2, E3, E4, are all considered having three influence factors: the value of $K$, demand $\left(d_{k}\right)$, and the penalty $\left(p_{k}\right)$. Table 3 clearly presents a summary of all experimental frameworks.

TABLE 2. RESULTS FOR EXPERIMENT E1

\begin{tabular}{cccccccccc}
\hline$K$ & $r$ & $C$ & result & time & $K$ & $r$ & $C$ & result & time \\
\hline \multirow{4}{*}{100} & & 0.2 & 216 & 63.5240 & & & 0.2 & 227 & 63.5390 \\
& & 0.4 & 227 & 63.8040 & & & 0.4 & 230 & 63.6790 \\
& & 0.5 & 234 & 63.6790 & 100 & 0.7 & 0.5 & 227 & 64.3810 \\
& & 0.7 & 223 & 63.4300 & & & 0.7 & 227 & 63.3680 \\
& 0.9 & 222 & 63.3050 & & & 0.9 & 222 & 63.3050 \\
\hline & & 0.2 & 226 & 63.8510 & & & 0.2 & 203 & 63.4490 \\
100 & 1.0 & 0.4 & 221 & 63.6500 & & & 0.4 & 215 & 63.5700 \\
& & 0.5 & 206 & 64.1160 & 100 & 1.2 & 0.5 & 227 & 63.9750 \\
& & 0.9 & 226 & 63.3990 & & & 0.7 & 225 & 63.2890 \\
& & 226 & 63.2110 & & & 0.9 & 236 & 63.0860 \\
\hline & & 0.2 & 234 & 63.4920 & & & & & \\
100 & 1.2 & 0.4 & 218 & 63.8660 & & & & & \\
& & 0.5 & 222 & 63.5540 & & & & & \\
& & 0.7 & 228 & 63.4140 & & & & & \\
& 0.9 & 229 & 63.0710 & & & & & \\
\hline
\end{tabular}

TABLE 3. FRAMEWORK OF COMPARISON EXPERIMENTS

\begin{tabular}{lccc}
\hline & $K$ & $d_{k}$ & $p_{k}$ \\
\hline$E_{2} 40,100,150,180,300,500,1000$ & $U[1,20]$ & $U[1,10]$ \\
$E_{3}$ & 100 & $U[20,50], U[1,100], U[100,200], U[100,800]$ & $U[1,20], U[20,50]$ \\
$E_{4}$ & 50,120 & $U[20,50]$ & $U[1,6], U[8,15], U[20,60], U[50,100]$ \\
\hline
\end{tabular}

TABLE 4. RESULTS FOR EXPERIMENT E2

\begin{tabular}{ccccccc}
\hline \multicolumn{3}{c}{ DPSO-GA } & \multicolumn{3}{c}{ DPSO-SA } \\
\hline$K$ & $d_{k}$ & $p_{k}$ & result & time & result & time \\
\hline 40 & & & 197 & 6.6450 & 193 & 16.0520 \\
100 & $U[1,20]$ & & 424 & 62.9300 & 473 & 63.2430 \\
150 & & 841 & 115.1810 & 854 & 116.5570 \\
180 & & $U[1,10]$ & 1130 & 152.7030 & 1019 & 155.8240 \\
300 & & & 1792 & 348.8240 & 1829 & 356.2110 \\
500 & & & 3166 & 824.7430 & 3450 & 858.1210 \\
1000 & & & 6827 & $3.0043 e+003$ & 6922 & $3.0153 e+003$ \\
\hline
\end{tabular}


TABLE 5. . . RESULTS FOR EXPERIMENT E3

\begin{tabular}{ccccccc}
\hline & & & DPSO-GA & \multicolumn{3}{c}{ DPSO-SA } \\
\hline \multirow{4}{*}{100} & $d_{k}$ & $p_{k}$ & result & time & result & time \\
\hline \multirow{4}{*}{100} & $U[20,50]$ & & 1407 & 62.7900 & 1330 & 63.4140 \\
& $U[1,100]$ & & 1328 & 62.3850 & 1465 & 63.3830 \\
& $U[100,200]$ & $U[1,20]$ & 2655 & 62.3530 & 3286 & 63.8360 \\
& $U[100,800]$ & & 4964 & 62.2590 & 7959 & 63.3050 \\
\hline & $U[20,50]$ & & 2995 & 62.1970 & 3079 & 63.4140 \\
& $U[1,100]$ & & 3171 & 62.2910 & 3042 & 63.3200 \\
& $U[100,200]$ & $U[20,50]$ & 4961 & 62.4320 & 4605 & 62.8360 \\
& & 7884 & 61.9480 & 8257 & 63.1490 \\
\hline
\end{tabular}

TABLE 6. . . RESULTS FOR EXPERIMENT E4

\begin{tabular}{|c|c|c|c|c|c|c|}
\hline & & & DPSO-G & & PSO-S & \\
\hline$K$ & $d_{k}$ & $p_{k}$ & result & time & result & time \\
\hline \multirow{4}{*}{50} & \multirow{4}{*}{$U[20,50]$} & $U[1,6]$ & 254 & 22.8540 & 257 & 22.5420 \\
\hline & & $U[8,15]$ & 673 & 23.1350 & 612 & 22.6510 \\
\hline & & $U[20,60]$ & 1566 & 23.1660 & 1571 & 22.4950 \\
\hline & & $U[50,100]$ & 1654 & 23.0720 & 1830 & 22.5730 \\
\hline \multirow{4}{*}{120} & \multirow{4}{*}{$U[20,50]$} & $U[1,6]$ & 1007 & 81.3380 & 969 & 82.5870 \\
\hline & & $U[8,15]$ & 1870 & 81.4170 & 1871 & 82.2440 \\
\hline & & $U[20,60]$ & 4017 & 81.2770 & 3992 & 82.9300 \\
\hline & & $U[50,100]$ & 4722 & 83.1630 & 5074 & 82.9920 \\
\hline
\end{tabular}

In table 4 , we can see that the all results obtained by the DPSO-SA algorithm is bigger than that of the DPSO-GA algorithm expect the case of value $K=180$, especially, the difference of result is 284 between the two DPSO algorithms, so the DPSO-GA is better to get the optimal result. In addition, the total time of DPSO-GA is shorter than that of the DPSO-SA, the comparison is the most outstanding at value $K=40$. But beyond that, there is a very obvious disadvantage both in the two DPSO algorithms that is the total time of the two DPSO algorithms will increase to about 3000 seconds which is about 50 minutes when the request calls is up to 1000 . So, the two algorithms will cost too much time to solve the actual problem when request calls is a large number.

Table 5 shows the results for experiment E3, and performs the changing fact of results and times when the demand increases. We can see that the result of DPSO-SA algorithm is more than that of DPSO-GA clearly once the demand increases to $U[100,800]$. However, there are two results bigger than that of the DPSO-SA, which show that the DPSO-GA algorithm is not stable.

In the Table 6, it is shown that the different results between DPSO-GA algorithm and DPAO-SA algorithm when the penalty increased. We can see that the influence of penalty's supremum is not be neglected, in which the results of the two algorithms are the most significant once the penalty's supremum is up to 100 . Therefore, the DPAO-GA is better to solve the PCCC problems with higher penalty.
Analysis of convergence. For more effective comparison on the two algorithm, we use their convergence to do it. First, we let three variables be $K=100, \quad d_{k} \in U[1,20], \quad p_{k} \in U[1,10]$, the first two pictures of Fig.1. performs the convergence that DPSO-GA has better convergence rate, in which the fit curve of DPSO-GA is in an iterative smooth before 30 times but the other is after 30times. Then we replace the value of $K$, let $K$ be 50 . The last two pictures of Fig. 1 show that the convergence of DPSO-GA begins to converge for 20 iterations, the other begins for 40 iterations. 


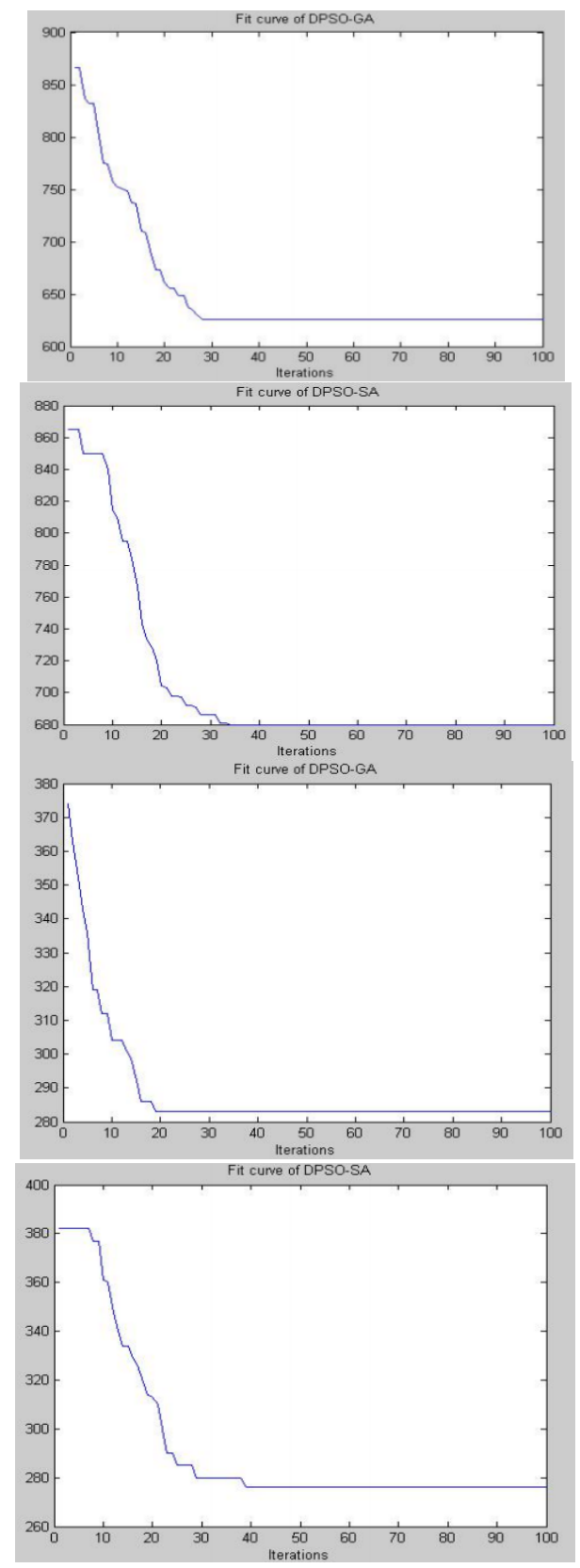

Figure 1. Fit curve with $K=100$ and $K=50$.

\section{CONCLUSION}

To our knowledge, the application of PSO algorithm in solving the PCCC problem on lines was proposed in this paper. We proposed two DPSO algorithms and then compare optimal results, running time and convergence of the two algorithms. Also, we found that DPSO-GA outperforms DPSO-SA in all of the experimental frameworks. We do some experiments to compare the results and iteration time of the two DPSO algorithms,_we found that it is not about good or bad about the two algorithms, it is all about certain point. In addition, the two DPSO algorithms is not perfect, which have many disadvantages, thus, finding preferable ways to solve the PCCC problems is interesting in the future. Also, extending our two DPSO algorithms for solving other problems is attractive.

\section{ACKNOWLEDGMENTS}

The research was supported by the National Nature Science Foundation of China [No. 11301466] and the Natural Science Foundation of Yunnan Province of China [No.2014FB114].

\section{REFERENCES}

[1]. N. Bansal, Z. Friggstad, R. Khandekar and M.R. Salavatipour.: A Logarithmic Approximation for Unsplittable Flow on Line Graphs. In: Proceeding of the Twentieth Annual ACM-SIAM Symposium on Discrete Algorithms, pp. 1-15 (2014)

[2]. P. Bonsma, J.Schulz and A. Wiese.: A Constant-factor Approximation Algorithm for Unsplit-table Flow on Paths. J. SIAM J. Compute. 43, 767-799 (2014)

[3]. A. Anagnostopoulos, F. Grandoni, S. Leonardi and A. Wiese.: A Mazing $2+\varepsilon$ Approximation for Unsplittable Flow on a Path. In: Proceeding of the Twenty-Fifth Annual ACM-SIAM Symposium on Discrete Algorithms, pp. 26-41 (2014)

[4]. J. Batra, N. Garg, A. Kumar, T. Mo"mek and A. Wiese.: New Approximation Schemes for Unsplittable Flow on a Path. In Proceeding of the Twenty-Sixth Annual ACM-SIAM Sym-posium on Discrete Algorithms, pp. 47-58 (2015)

[5]. A. Bar-Noy, R. Bar-Yehuda, A. Freund, J. Naor and B. Schieber.: A Unified Approach to Approximating Resource Allocation and Scheduling. J. ACM. 48, 1069-1090 (2001)

[6]. W. Li, J. Li, L. Guan and Y. Shi.: The Parize-Collection Call Control Problem on Weighted Lines and Rings. J. Rai. Oper. Rea. $50,39-46(2016)$

[7]. W. Li, J. Li, L. Guan.: Approximation Algorithms for the Ring Loading Problem with Penalty Cost. J. Inf. Pro. Let. 114, 56-59 (2014)

[8]. A. Archer, M.H. Beteni, M.T. Hajianhayi and H. Karloff.: Improved Approximation Algo-rithms for Prize-Collecting Steiner Tree and TSP. J. SIAM J. Cmpute. 40, 309-332 (2011)

[9]. J. Kennedy, RC. Eberhart.: Particle Swarm Optimization. In: Proceeding of IEEE International Conference on Neural Networks, pp. 1942-1948. IEEE Serverce Center, Piscataway (1995)

[10]. A. H. Kashan and B. Karimi.: A Discrete Particle Swarm Optimization Algorithm for Scheduling Parallel Machines. J. Comprter and Industrial Engineering. 56, 216-223 (2009)

[11]. N. Metropolis, AW. Rosenbluth, MN. Rosenbluth, AH. Teller and E. Teller.: Equation of State Calculations by Fast Computing Machines. J. Chem. Phys. 21, 1087-1090 (1953) 